\title{
GLOBAL DYNAMICS OF THE INTEGRABLE ARMBRUSTER-GUCKENHEIMER-KIM GALACTIC POTENTIAL
}

\author{
JAUME LLIBRE AND CLAUDIA VALLS
}

\begin{abstract}
We study the global dynamics of the completely integrable Armbruster-Guckenheimer-Kim galactic potential. In these cases this system has two first integrals $H_{1}$ and $H_{2}$ independent and in involution. Let $I_{h_{1}}$ and $I_{h_{2}}$ be the set of points of the phase space on which $H_{1}$ and $H_{2}$ take the values $h_{1}$ and $h_{2}$, respectively. The sets $I_{h_{1} h_{2}}=I_{h_{1}} \cap I_{h_{2}}$ are invariant by the dynamics. We characterize the global flow on these sets and we describe the foliation of the phase space by the invariant sets $I_{h_{1} h_{2}}$.
\end{abstract}

\section{INTRODUCTION}

The Armsbruster-Guckenheimer-Kim potential is a galactic potential introduced in [2] that studies the dynamics for the interchanging of nearly nondegenerate modes with square symmetry. They derived the model starting with a normal form given by a system of differential equations which represented the codimension two bifurcation problem. More precisely, the Hamiltonian function that they provided is

$$
H\left(x, p_{x}, y, p_{y}\right)=\frac{1}{2}\left(p_{x}^{2}+p_{y}^{2}\right)+\frac{1}{2}\left(x^{2}+y^{2}\right)-\frac{a}{4}\left(x^{2}+y^{2}\right)^{2}-\frac{b}{2} x^{2} y^{2},
$$

where $a, b$ are arbitrary constants. If we add the term $-\omega\left(x p_{y}-y p_{x}\right)$ then the system describes the dynamics of rotation of a nearly axisymetric galaxy rotating with a constant velocity $\omega$ around a fixed axis. The existence of such $\omega$ denotes that the rotation of the galaxy must be taken into account when we study the stellar orbits (see [8]). Many studies concerning the integrability and non-integrability of such systems have been done (see for instance $[1,4,5]$ ) using different techniques such as the Painlevé analysis and the Morales-Ramis theory as well as the study of the existence of periodic orbits which was done in [7]. In particular, it was proved in [5] that if $b=2 a$ or $b=-a$ the

Key words and phrases. Armbruster-Guckenheimer-Kim galactic potential, invariant manifolds, complete integrability. 
system is completely integrable but the authors do not describe completely the dynamics of the integrable systems form the point of view of the Liouville-Arnold theorem (see section 2). This is the main aim of this paper.

When $b=2 a$ the Hamiltonian has the form

$$
H=\frac{1}{2}\left(p_{x}^{2}+p_{y}^{2}\right)+\frac{1}{2}\left(x^{2}+y^{2}\right)-\frac{a}{4}\left(x^{2}+y^{2}\right)^{2}-a x^{2} y^{2} .
$$

Introducing the new variables

$u=\frac{1}{\sqrt{2}}(x-y), v=\frac{1}{\sqrt{2}}(x+y), p_{u}=\frac{1}{\sqrt{2}}\left(p_{x}-p_{y}\right), p_{v}=\frac{1}{\sqrt{2}}\left(p_{x}+p_{y}\right)$,

it can be written as

$$
\begin{aligned}
H\left(x, p_{x}, y, p_{y}\right) & =\frac{1}{2}\left(p_{x}^{2}+p_{y}^{2}\right)+\frac{a}{4}\left(x^{4}+y^{4}\right)-\frac{1}{2}\left(x^{2}+y^{2}\right) \\
& =\tilde{H}_{1}\left(x, p_{x}\right)+\tilde{H}_{2}\left(y, p_{y}\right),
\end{aligned}
$$

where $a \in \mathbb{R}$, we have renamed the variables $(u, v)$ again as $(x, y)$ and

$$
\tilde{H}_{1}\left(x, p_{x}\right)=\frac{1}{2} p_{x}^{2}+\frac{a}{4} x^{4}-\frac{1}{2} x^{2}, \quad \tilde{H}_{2}\left(y, p_{y}\right)=\frac{1}{2} p_{y}^{2}+\frac{a}{4} y^{4}-\frac{1}{2} y^{2} .
$$

Note that $\tilde{H}_{i}: \mathbb{R}^{2} \rightarrow \mathbb{R}$ while $H: \mathbb{R}^{4} \rightarrow \mathbb{R}$. In all the paper we will denote by $H$ the Hamiltonian associated to a system with two degrees of freedom and so $H=H\left(x, p_{x}, y, p_{y}\right): \mathbb{R}^{4} \rightarrow \mathbb{R}, H_{i}=H_{i}\left(x, p_{x}, y, p_{y}\right): \mathbb{R}^{4} \rightarrow$ $\mathbb{R}$ for $i=1, \ldots, 4$, and we will denote by $\tilde{H}$ the Hamiltonian associated to a system with one degree of freedom and so $\tilde{H}_{1}=\tilde{H}_{1}\left(x, p_{x}\right): \mathbb{R}^{2} \rightarrow \mathbb{R}$ and $\tilde{H}_{2}=\tilde{H}_{2}\left(y, p_{y}\right): \mathbb{R}^{2} \rightarrow \mathbb{R}$.

We observe thta $H_{1}$ and $H_{2}$ are two first integrals, independent and in involution. Hence, the Hamiltonian system associated to the Hamiltonian $H$ is

$$
\dot{x}=p_{x}, \quad \dot{y}=p_{y}, \quad \dot{p}_{x}=-a x^{3}+x, \quad \dot{p}_{y}=-a y^{3}+y
$$

and it is completely integrable. We recall that $H_{1}$ and $H_{2}$ are independent if the matrix

$$
\left(\begin{array}{llll}
H_{1 x} & H_{1 p_{x}} & H_{1 y} & H_{1 p_{x}} \\
H_{2 x} & H_{2 p_{x}} & H_{2 y} & H_{2 p_{x}}
\end{array}\right)
$$

has rank 2 in any point of $\mathbb{R}^{4}$ except, perhaps in a zero Lebesguemeasure set. As usual $H_{i y}=\partial H_{i} / \partial y$. Moreover, we say that $H_{1}$ and $H_{2}$ are in involution if their Poisson bracket is zero. Finally, a Hamiltonian system with two degrees of freedom is completely integrable if it has two independent first integrals in involution. 
Note that the phase space of system (1) is $\mathbb{R}^{4}$. Since $H_{1}$ and $H_{2}$ are fist integrals the sets

$$
\begin{aligned}
I_{h_{1}} & =\left\{\left(x, p_{x}, y, p_{y}\right) \in \mathbb{R}^{4}: H_{1}=h_{1}\right\}=\left\{\left(x, p_{x}\right) \in \mathbb{R}^{2}: \tilde{H}_{1}=\tilde{h}_{1}\right\} \times \mathbb{R}^{2} \\
& =I_{\tilde{h}_{1}} \times \mathbb{R}^{2}, \\
I_{h_{2}} & =\left\{\left(x, p_{x}, y, p_{y}\right) \in \mathbb{R}^{4}: H_{2}=h_{2}\right\}=\left\{\left(y, p_{y}\right) \in \mathbb{R}^{2}: \tilde{H}_{2}=\tilde{h}_{2}\right\} \times \mathbb{R}^{2} \\
& =\mathbb{R}^{2} \times I_{\tilde{h}_{2}},
\end{aligned}
$$

as well as

$$
\begin{aligned}
I_{h_{1} h_{2}} & =\left\{\left(x, p_{x}, y, p_{y}\right) \in \mathbb{R}^{4}: H_{1}=h_{1}, H_{2}=h_{2}\right\} \\
& =\left\{\left(x, p_{x}, y, p_{y}\right) \in \mathbb{R}^{4}: H_{1}=h_{1}\right\} \cap\left\{\left(x, p_{x}, y, p_{y}\right) \in \mathbb{R}^{4}: H_{2}=h_{2}\right\} \\
& =I_{h_{1}} \cap I_{h_{2}}=\left(I_{\tilde{h}_{1}} \times \mathbb{R}^{2}\right) \cap\left(\mathbb{R}^{2} \times I_{\tilde{h}_{2}}\right) \\
& =I_{\tilde{h}_{1}} \times I_{\tilde{h}_{2}}
\end{aligned}
$$

are invariant by the flow of the Hamiltonian system (1). The first objective of this paper is to describe the foliations of the phase space $\mathbb{R}^{4}$ by the invariant sets $I_{h_{i}}$ for $i=1,2$ as well as by $I_{h_{1} h_{2}}$. The foliations provide a good description of the phase portraits of the Hamiltonian flow (1) when $a$ varies.

When $b=-a$ the Hamiltonian has the form

$$
\begin{aligned}
H\left(x, p_{x}, y, p_{y}\right) & =\frac{1}{2}\left(p_{x}^{2}+p_{y}^{2}\right)-\frac{a}{4}\left(x^{4}+y^{4}\right)+\frac{1}{2}\left(x^{2}+y^{2}\right) \\
& =\tilde{H}_{3}\left(x, p_{x}\right)+\tilde{H}_{4}\left(y, p_{y}\right),
\end{aligned}
$$

where $a \in \mathbb{R}$ with

$$
\tilde{H}_{3}\left(x, p_{x}\right)=\frac{1}{2} p_{x}^{2}-\frac{a}{4} x^{4}+\frac{1}{2} x^{2}, \quad \tilde{H}_{4}\left(y, p_{y}\right)=\frac{1}{2} p_{y}^{2}-\frac{a}{4} y^{4}+\frac{1}{2} y^{2} .
$$

Note that $H_{3}$ and $H_{4}$ are two first integrals, independent and in involution. Hence the Hamiltonian system

$$
\dot{x}=p_{x}, \quad \dot{y}=p_{y}, \quad \dot{p}_{x}=a x^{3}-x, \quad \dot{p}_{y}=a y^{3}-y
$$

is completely integrable. The sets

$$
\begin{aligned}
& I_{h_{3}}=\left\{\left(x, p_{x}, y, p_{y}\right) \in \mathbb{R}^{4}: H_{3}=h_{3}\right\}=I_{\tilde{h}_{3}} \times \mathbb{R}^{2}, \\
& I_{h_{4}}=\left\{\left(x, p_{x}, y, p_{y}\right) \in \mathbb{R}^{4}: H_{4}=h_{4}\right\}=\mathbb{R}^{2} \times I_{\tilde{h}_{3}},
\end{aligned}
$$

as well as

$$
I_{h_{3} h_{4}}=\left\{\left(x, p_{x}, y, p_{y}\right) \in \mathbb{R}^{4}: H_{3}=h_{3}, H_{4}=h_{4}\right\}=I_{h_{3}} \cap I_{h_{4}}=I_{\tilde{h}_{3}} \times I_{\tilde{h}_{4}}
$$

are invariant by the flow of the Hamiltonian system (2). The second main objective of the paper is to describe the foliations of $\mathbb{R}^{4}$ by the invariant sets $I_{h_{i}}$ for $i=3,4$ and by the invariant sets $I_{h_{3} h_{4}}$. Again, 
these foliations provide a good description of the phase portraits of the Hamiltonian flow (2) when $a$ varies.

The paper is organized as follows. In section 2 we recall the LiouvilleArnold theory for Hamiltonians systems with two degrees of freedom. In section 3 we describe the topology of the sets $I_{h_{1}}$ (since the study for $I_{h_{2}}$ is analogous). For doing that and taking into account that $I_{h_{1}}=I_{\tilde{h}_{1}} \times \mathbb{R}^{2}$ we will only describe the topology of the sets $I_{\tilde{h}_{1}}$ by computing the sets of singular points and critical values for $\tilde{H}_{1}$ and the Hill regions according to the different values of $a$ and $\tilde{h}_{1}$. In section 4 we study the topology of the sets $I_{h_{1} h_{2}}$. In section 5 we describe the topology of the sets $I_{h_{3}}$ (again because the study for $I_{h_{4}}$ is analogous) and recalling that $I_{h_{3}}=I_{\tilde{h}_{3}} \times \mathbb{R}^{2}$ we will only describe the topology of the sets $I_{\tilde{h}_{3}}$ by computing the sets of singular points and critical values for $\tilde{H}_{3}$ and the Hill regions according to the different values of $a$ and $\tilde{h}_{3}$. In section 6 we study the topology of the sets $I_{h_{3} h_{4}}$.

\section{Integrable HAMiltonian SYSTEMS}

In this section we recall the Liouville-Arnold theorem for the integrable Hamiltonian systems with two degrees of freedom. We recall that a flow defined on the phase space $\mathbb{R}^{4}$ is complete if its solutions are defined for all time $t$ in $\mathbb{R}$.

Theorem 1. The Hamiltonian system (1) (resp. system (2)) defined on the phase space $\mathbb{R}^{4}$ has the Hamiltonians $H_{1}$ and $H_{2}$ (resp. $H_{3}$ and $H_{4}$ ) as two independent first integrals in involution. If $I_{h_{1} h_{2}} \neq \emptyset$ (resp. $I_{h_{3} h_{4}} \neq \emptyset$ ) and $\left(h_{1}, h_{2}\right)$ (resp. $\left.\left(h_{3}, h_{4}\right)\right)$ is a regular value of the map $\left(H_{1}, H_{2}\right)$ (resp. $\left(H_{3}, H_{4}\right)$ ) then the following statements hold.

(a) $I_{h_{1} h_{2}}$ (resp. $\left.I_{h_{3} h_{4}}\right)$ is a two-dimensional submanifold of $\mathbb{R}^{4}$ invariant under the flow of system (1) (resp. system (2)).

(b) If the flow on a connected component $I_{h_{1} h_{2}}^{*}$ (resp. $\left.I_{h_{3} h_{4}}^{*}\right)$ of $I_{h_{1} h_{2}}$ (resp. $\left.I_{h_{3} h_{4}}\right)$ is complete, then $I_{h_{1} h_{2}}^{*}\left(\right.$ resp. $\left.I_{h_{3} h_{4}}^{*}\right)$ is diffeomorphic either to the torus $\mathbb{S}^{1} \times \mathbb{S}^{1}$, to the cylinder $\mathbb{S}^{1} \times \mathbb{R}$, or to the plane $\mathbb{R}^{2}$

(c) Under the assumption of statement (b), the flow on $I_{h_{1} h_{2}}^{*}$ (resp. on $\left.I_{h_{3} h_{4}}^{*}\right)$ is conjugated to a linear flow either on $\mathbb{S}^{1} \times \mathbb{S}^{1}$, or on $\mathbb{S}^{1} \times \mathbb{R}$, or on $\mathbb{R}^{2}$.

Note that Theorem 1 does not provide information on the topology of the invariant sets $I_{h_{1} h_{2}}\left(\operatorname{resp} . I_{h_{3} h_{4}}\right)$ when $\left(h_{1} h_{2}\right)\left(\operatorname{resp} .\left(h_{3} h_{4}\right)\right)$ is 
not a regular value of the map $\left(H_{1}, H_{2}\right)$ (resp. $\left.\left(H_{3}, H_{4}\right)\right)$, or how the energy levels $I_{h_{1}}$ or $I_{h_{2}}$ (resp. $I_{h_{3}}$ or $I_{h_{4}}$ ) foliate $\mathbb{R}^{4}$.

In this paper we solve these problems for systems (1) and (2).

\section{The TOPOLOGY OF THE INVARIANT SETS $I_{h_{1}}$}

As explained in the introduction, taking into account that $I_{h_{1}}=$ $I_{\tilde{h}_{1}} \times \mathbb{R}^{2}$ we will restrict all the study to $I_{\tilde{h}_{1}}$.

A point $\left(x, p_{x}\right) \in \mathbb{R}^{2}$ is a singular point for the map $\tilde{H}_{1}$ if it is a solution of

$$
\frac{\partial \tilde{H}_{1}}{\partial p_{x}}=0, \quad \frac{\partial \tilde{H}_{1}}{\partial x}=0
$$

The value $\tilde{h}_{1} \in \mathbb{R}$ is a critical value for the map $\tilde{H}_{1}$ if there is some singular point belonging to $\tilde{H}_{1}^{-1}\left(\tilde{h}_{1}\right)=I_{\tilde{h}_{1}}$. If $\tilde{h}_{1}$ is not critical value it is said a regular value. It is well-known that if $\tilde{h}_{1}$ is a regular value of the map $\tilde{H}_{1}$ then $I_{\tilde{h}_{1}}$ is a one-dimensional manifold (see [6]).

Note that the singular points for the map $\tilde{H}_{1}$ are

$$
p_{x}=0, \quad x\left(a x^{2}-1\right)=0,
$$

and so the set of singular points of $\tilde{H}_{1}$ is $(0,0)$ if $a \leq 0$, and $(0,0) \cup$ $(0,-1 / \sqrt{a}) \cup(0,1 / \sqrt{a})$ if $a>0$.

We define the Hill region as

$$
R_{\tilde{h}_{1}}=\left\{x \in \mathbb{R}: \frac{a}{4} x^{4}-\frac{x^{2}}{2} \leq \tilde{h}_{1}\right\}
$$

This is the region of the configuration space $\{x \in \mathbb{R}\}$ where the motion of all orbits of the Hamiltonian system associated to $\tilde{H}_{1}$ having energy $\tilde{h}_{1}$ takes place. By $R_{\tilde{h}_{1}} \approx S$, we denote that $R_{\tilde{h}_{1}}$ is diffeomorphic to $S$. We will also denote by

$$
P_{-}=\sqrt{\frac{1-\sqrt{1+4 a \tilde{h}_{1}}}{a}}, \quad P_{+}=\sqrt{\frac{1+\sqrt{1+4 a \tilde{h}_{1}}}{a}}
$$

have:

(i) $R_{\tilde{h}_{1}} \approx \mathbb{R}$ if $a=0$ and $\tilde{h}_{1}>0$,

(ii) $R_{\tilde{h}_{1}} \approx \mathbb{R}$ but here $\{0\}$, which is a singular point for $\tilde{H}_{1}$, is in the boundary of the Hill region, if $a=0$ and $\tilde{h}_{1}=0$,

(iii) $R_{\tilde{h}_{1}} \approx\left(-\infty,-\sqrt{-2 \tilde{h}_{1}}\right] \cup\left[\sqrt{-2 \tilde{h}_{1}}, \infty\right)$ if $a=0$ and $\tilde{h}_{1}<0$,

(iv) $R_{\tilde{h}_{1}} \approx \mathbb{R}$ if $a<0$ and $\tilde{h}_{1}>0$, 
(v) $R_{\tilde{h}_{1}} \approx \mathbb{R}$ but here $\{0\}$, which is a singular point for $\tilde{H}_{1}$, is in the boundary of the Hill region, if $a<0$ and $\tilde{h}_{1}=0$,

(vi) $R_{\tilde{h}_{1}} \approx\left(-\infty,-P_{-}\right] \cup\left[P_{-}, \infty\right)$ if $a<0$ and $\tilde{h}_{1}<0$,

(vii) $R_{\tilde{h}_{1}} \approx \emptyset$ if $a>0$ and $\tilde{h}_{1}<-1 /(4 a)$,

(viii) $R_{\tilde{h}_{1}} \approx\left\{-\sqrt{\frac{1}{a}}\right\} \cup\left\{\sqrt{\frac{1}{a}}\right\}$ which are two of the singular points for the map $\tilde{H}_{1}$, if $a>0$ and $\tilde{h}_{1}=-1 /(4 a)$,

(ix) $R_{\tilde{h}_{1}} \approx\left[-P_{+},-P_{-}\right] \cup\left[P_{-}, P_{+}\right]$, if $a>0$ and $\tilde{h}_{1} \in(-1 /(4 a), 0)$,

(x) $R_{\tilde{h}_{1}} \approx\left[-\sqrt{\frac{2}{a}}, \sqrt{\frac{2}{a}}\right]$ but here $\{0\}$, which is a singular point for $\tilde{H}_{1}$, is in the boundary of the Hill region, if $a>0$ and $\tilde{h}_{1}=0$,

(xi) $R_{\tilde{h}_{1}} \approx\left[-P_{+}, P_{+}\right]$if $a>0$ and $\tilde{h}_{1}>0$. have

Now we compute the energy levels $I_{\tilde{h}_{1}}$. From the definition of $I_{\tilde{h}_{1}}$ we

$$
I_{\tilde{h}_{1}}=\bigcup_{x \in R_{\tilde{h}_{1}}} E_{x}
$$

where

$$
E_{x}=\left\{\left(x, p_{x}\right) \in \mathbb{R}^{2}: \frac{p_{x}^{2}}{2}+\frac{a}{4} x^{4}-\frac{1}{2} x^{2}=\tilde{h}_{1}\right\} .
$$

Clearly for each $x \in \mathbb{R}$ the set $E_{x}$ is either two points, or one point or the emptyset, if the point $x$ is in the interior of the Hill region $R_{\tilde{h}_{1}}$, in its boundary, or it does not belong to $R_{\tilde{h}_{1}}$, respectively. Therefore, from (3) and using the Hill region, the topology of $I_{\tilde{h}_{1}}$ is:

(i) $I_{\tilde{h}_{1}} \approx \mathbb{R} \cup \mathbb{R}$ if $a<0$ and $\tilde{h}_{1} \neq 0$,

(ii) $I_{\tilde{h}_{1}} \approx X$ if $a \leq 0$ and $\tilde{h}_{1}=0$. Here $X$ denotes two straight lines intersecting the origin of the two straight lines,

(iii) $I_{\tilde{h}_{1}} \approx \emptyset$ if $a>0$ and $\tilde{h}_{1}<-1 /(4 a)$,

(iv) $I_{\tilde{h}_{1}} \approx\left( \pm \sqrt{\frac{1}{a}}, 0\right)$ which are the two equilibrium points of $\tilde{H}_{1}$ if $a>0$ and $\tilde{h}_{1}=-1 /(4 a)$,

(v) $I_{\tilde{h}_{1}} \approx \mathbb{S}^{1} \cup \mathbb{S}^{1}$ if $a>0$ and $\tilde{h}_{1} \in(-1 /(4 a), 0)$,

(vi) $I_{\tilde{h}_{1}} \approx \infty$ if $a>0$ and $\tilde{h}_{1}=0$. Here $\infty$ denotes two homoclinic orbits at the origin.

(vii) $I_{\tilde{h}_{1}} \approx \mathbb{S}^{1}$ if $a>0$ and $\tilde{h}_{1}<0$.

See in Figure 1 the phase portraits associated to the Hamiltonian system with Hamiltonian $\tilde{H}_{1}$ depending on whether $a>0, a=0$, and $a<0$. The phase portraits in Figure 1 are drawn in the Poincaré disc, which essentially is a unit closed disc centered at the origin of 
coordinates with its interior identified to $\mathbb{R}^{2}$ and with its boundary (the circle $\mathbb{S}^{1}$ ) identified with the infinity of $\mathbb{R}^{2}$, for more details on the Poincaré disc see Chapter 5 of [3].

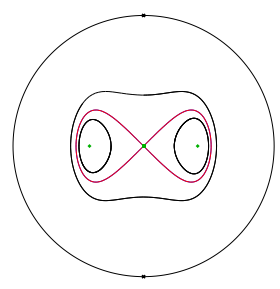

(a) $a>0$

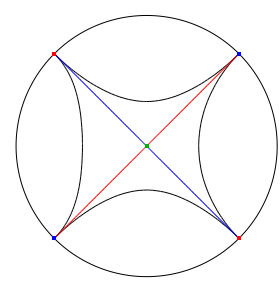

(b) $a=0$

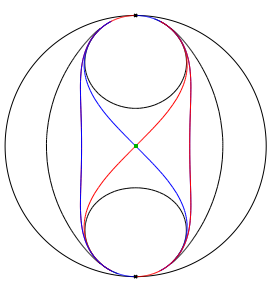

(c) $a<0$

FiguRE 1. Phase portraits associated to the Hamiltonian system with Hamiltonian $\tilde{H}_{1}$ depending on whether $a>0, a=0$ and $a<0$.

\section{The topology of THE InVARIANT SETS $I_{h_{1} h_{2}}$}

To obtain $I_{h_{1} h_{2}}$ we recall that $I_{h_{2}}$ is exactly the same as $I_{h_{1}}$ and that $I_{h_{1} h_{2}}=I_{h_{1}} \cap I_{h_{2}}=I_{\tilde{h}_{1}} \times I_{\tilde{h}_{2}}$. Hence, in Table 1 we have given the description of the invariant sets $I_{h_{1} h_{2}}$ for the different values of $h_{1}, h_{2}$ and $a$

\section{The TOPOLOGY OF THE INVARIANT SETS $I_{h_{3}}$}

As we did for the case $H_{1}$, we recall that $I_{h_{3}}=I_{\tilde{h}_{3}} \times \mathbb{R}^{2}$ and so we will study only $I_{\tilde{h}_{3}}$. The singular points for the map $\tilde{H}_{3}$ satisfy

$$
p_{x}=0, \quad x\left(1-a x^{2}\right)=0
$$

and so they are $(0,0)$ if $a \leq 0$ and $(0,0) \cup(0,-1 / \sqrt{a}) \cup(0,1 / \sqrt{a})$ if $a>0$. The Hill region is

$$
R_{\tilde{h}_{3}}=\left\{y \in \mathbb{R}:-\frac{a}{4} y^{4}+\frac{y^{2}}{2} \leq \tilde{h}_{3}\right\}
$$

and so taking the notation

$$
Q_{-}=\sqrt{\frac{1-\sqrt{1-4 a \tilde{h}_{3}}}{a}}, \quad Q_{+}=\sqrt{\frac{1+\sqrt{1-4 a \tilde{h}_{3}}}{a}}
$$

we have

(i) $R_{\tilde{h}_{3}} \approx \emptyset$ if $a=0$ and $\tilde{h}_{3}<0$, 


\begin{tabular}{|c|c|c|c|}
\hline$a$ & $h_{1}$ & $h_{2}$ & $I_{h_{1} h_{2}}$ \\
\hline$\leq 0$ & $\neq 0$ & $\neq 0$ & $(\mathbb{R} \cup \mathbb{R}) \times(\mathbb{R} \cup \mathbb{R})$ \\
\hline$\leq 0$ & $\neq 0$ & $=0$ & $(\mathbb{R} \cup \mathbb{R}) \times X$ \\
\hline$\leq 0$ & $=0$ & $\neq 0$ & $X \times(\mathbb{R} \cup \mathbb{R})$ \\
\hline$\leq 0$ & $=0$ & $=0$ & $X \times X$ \\
\hline$>0$ & $<-1 /(4 a)$ & $\in \mathbb{R}$ & $\emptyset$ \\
\hline$>0$ & $=-1 /(4 a)$ & $<-1 /(4 a)$ & $\emptyset$ \\
\hline$>0$ & $=-1 /(4 a)$ & $=-1 /(4 a)$ & $\left( \pm \sqrt{\frac{1}{a}}, 0\right) \times\left( \pm \sqrt{\frac{1}{a}}, 0\right)$ \\
\hline$>0$ & $=-1 /(4 a)$ & $\in(-1 /(4 a), 0)$ & $\left( \pm \sqrt{\frac{1}{a}}, 0\right) \times\left(\mathbb{S}^{1} \cup \mathbb{S}^{1}\right)$ \\
\hline$>0$ & $=-1 /(4 a)$ & $=0$ & $\left( \pm \sqrt{\frac{1}{a}}, 0\right) \times \infty$ \\
\hline$>0$ & $=-1 /(4 a)$ & $<0$ & $\left( \pm \sqrt{\frac{1}{a}}, 0\right) \times \mathbb{S}^{1}$ \\
\hline$>0$ & $\in(-1 /(4 a), 0)$ & $<-1 /(4 a)$ & $\emptyset$ \\
\hline$>0$ & $\in(-1 /(4 a), 0)$ & $=-1 /(4 a)$ & $\left(\mathbb{S}^{1} \cup \mathbb{S}^{1}\right) \times\left( \pm \sqrt{\frac{1}{a}}, 0\right)$ \\
\hline$>0$ & $\in(-1 /(4 a), 0)$ & $\in(-1 /(4 a), 0)$ & $\left(\mathbb{S}^{1} \cup \mathbb{S}^{1}\right) \times\left(\mathbb{S}^{1} \cup \mathbb{S}^{1}\right)$ \\
\hline$>0$ & $\in(-1 /(4 a), 0)$ & $=0$ & $\left(\mathbb{S}^{1} \cup \mathbb{S}^{1}\right) \times \infty$ \\
\hline$>0$ & $\in(-1 /(4 a), 0)$ & $<0$ & $\left(\mathbb{S}^{1} \cup \mathbb{S}^{1}\right) \times \mathbb{S}^{1}$ \\
\hline$>0$ & $=0$ & $<-1 /(4 a)$ & $\emptyset$ \\
\hline$>0$ & $=0$ & $=-1 /(4 a)$ & $\infty \times\left( \pm \sqrt{\frac{1}{a}}, 0\right)$ \\
\hline$>0$ & $=0$ & $\in(-1 /(4 a), 0)$ & $\infty \times\left(\mathbb{S}^{1} \cup \mathbb{S}^{I}\right)$ \\
\hline$>0$ & $=0$ & $=0$ & $\infty \times \infty$ \\
\hline$>0$ & $=0$ & $<0$ & $\infty \times \mathbb{S}^{1}$ \\
\hline$>0$ & $<0$ & $<-1 /(4 a)$ & $\emptyset$ \\
\hline$>0$ & $<0$ & $=-1 /(4 a)$ & $\mathbb{S}^{1} \times\left( \pm \sqrt{\frac{1}{a}}, 0\right)$ \\
\hline$>0$ & $<0$ & $\in(-1 /(4 a), 0)$ & $\mathbb{S}^{1} \times\left(\mathbb{S}^{1} \cup \mathbb{S}^{1}\right)$ \\
\hline$>0$ & $<0$ & $=0$ & $\mathbb{S}^{1} \times \infty$ \\
\hline$>0$ & $<0$ & $<0$ & $\mathbb{S}^{1} \times \mathbb{S}^{1}$ \\
\hline
\end{tabular}

TABLE 1 . The invariant sets $I_{h_{1} h_{2}}$ for the different values of $h_{1}, h_{2}$ and $a$

(ii) $R_{\tilde{h}_{3}} \approx\{0\}$ if $a=0$ and $\tilde{h}_{3}=0$, then

(iii) $R_{\tilde{h}_{3}} \approx\left[-\sqrt{2 \tilde{h}_{3}}, \sqrt{2 \tilde{h}_{3}}\right]$ if $a=0$ and $\tilde{h}_{3}>0$ then

(iv) $R_{\tilde{h}_{3}} \approx \emptyset$ if $a<0$ and $\tilde{h}_{3}<0$,

(v) $R_{\tilde{h}_{3}} \approx\{0\}$ if $a<0$ and $\tilde{h}_{3}=0$,

(vi) $R_{\tilde{h}_{3}} \approx\left[-Q_{+}, Q_{-}\right]$if $a<0$ and $\tilde{h}_{3}>0$,

(vii) $R_{\tilde{h}_{3}} \approx \mathbb{R}$ if $a>0$ and $h_{3}>1 /(4 a)$, 
(viii) $R_{\tilde{h}_{3}} \approx \mathbb{R}$, but here $\left\{ \pm \sqrt{\frac{1}{a}}\right\}$, which are singular points for $\tilde{H}_{1}$, are in the boundary of the Hill region, if $a>0$ and $\tilde{h}_{3}=1 /(4 a)$,

(ix) $R_{\tilde{h}_{3}} \approx\left(-\infty,-Q_{+}\right] \cup\left[-Q_{-}, Q_{-}\right] \cup\left[Q_{+},+\infty\right)$ if $a>0$ and $\tilde{h}_{3} \in$ $(0,1 /(4 a))$

(x) $R_{\tilde{h}_{3}} \approx \mathbb{R}$, but here $\{0\}$, which is a singular point for $\tilde{H}_{1}$, is in the boundary of the Hill region, if $a>0$ and $\tilde{h}_{3}=0$,

(xi) $R_{\tilde{h}_{3}} \approx\left(-\infty,-Q_{+}\right] \cup\left[Q_{+},+\infty\right)$ if $a>0$ and $\tilde{h}_{3}<0$. have

Now we compute the energy levels $I_{\tilde{h}_{3}}$. From the definition of $I_{\tilde{h}_{3}}$ we

$$
I_{\tilde{h}_{3}}=\cup_{y \in R_{\tilde{h}_{3}}} E_{y}
$$

where

$$
E_{y}=\left\{\left(y, p_{y}\right) \in \mathbb{R}^{2}: \frac{p_{y}^{2}}{2}-\frac{a}{4} y^{4}+\frac{1}{2} y^{2}=\tilde{h}_{3}\right\} .
$$

Clearly for each $y \in \mathbb{R}$ the set $E_{y}$ is either two points, or one point or the emptyset, if the point $y$ is in the interior of the Hill region $R_{\tilde{h}_{3}}$, in its boundary, or it does not belong to $R_{\tilde{h}_{3}}$, respectively. Therefore, from (4) and using the Hill region, the topology of $I_{\tilde{h}_{3}}$ is:

(i) $I_{\tilde{h}_{3}} \approx \emptyset$ if $a \leq 0$ and $\tilde{h}_{3}<0$,

(ii) $I_{\tilde{h}_{3}} \approx\{(0,0)\}$ if $a \leq 0$ and $\tilde{h}_{3}=0$,

(iii) $I_{\tilde{h}_{3}} \approx \mathbb{S}^{1} a \leq 0$ and $\tilde{h}_{3}>0$,

(iv) $I_{\tilde{h}_{3}} \approx \mathbb{R} \cup \mathbb{R}$ if $a>0$ and $\tilde{h}_{3}>1 /(4 a)$,

(v) $I_{\tilde{h}_{3}} \approx P$ if $a>0$ and $\tilde{h}_{3}=1 /(4 a)$. Here $P$ denotes two curves with the shape of a parabola intersecting in two different points (the points are the two singular points),

(vi) $I_{\tilde{h}_{3}} \approx \mathbb{R} \cup \mathbb{S}^{1} \cup \mathbb{R}$ if $a>0$ and $\tilde{h}_{3} \in(0,1 /(4 a))$,

(vii) $I_{\tilde{h}_{3}} \approx \mathbb{R} \cup\{(0,0)\} \cup \mathbb{R}$ if $a>0$ and $\tilde{h}_{3}=0$,

(viii) $I_{\tilde{h}_{3}} \approx \mathbb{R} \cup \mathbb{R}$ if $a>0$ and $\tilde{h}_{3}<0$.

See the phase portrait associated to $\tilde{H}_{3}$ depending on whether $a>0$, $a=0$, or $a<0$.

See in Figure 2 the phase portraits associated to the Hamiltonian system with Hamiltonian $\tilde{H}_{3}$ depending on whether $a>0$ and $a \leq 0$.

\section{The TOPOLOGY OF THE INVARIANT SETS $I_{h_{3} h_{4}}$}

To obtain $I_{h_{3} h_{4}}$ we recall that $I_{h_{4}}$ is exactly the same as $I_{h_{3}}$ and that $I_{h_{3} h_{4}}=I_{h_{3}} \cap I_{h_{4}}=I_{\tilde{h}_{3}} \times I_{\tilde{h}_{4}}$. Hence, in Table 2 we have given the 


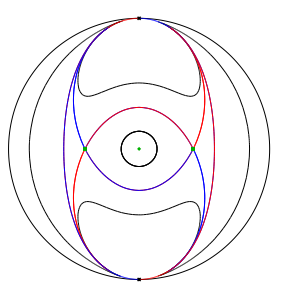

(a) $a>0$

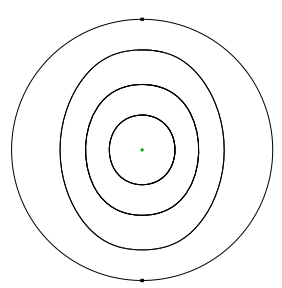

(b) $a \leq 0$

Figure 2. Phase portraits associated to the Hamiltonian system with Hamiltonian $\tilde{H}_{3}$ depending on whether $a>0$ or $a \leq 0$.

description of the invariant sets $I_{h_{3} h_{4}}$ for the different values of $h_{3}, h_{4}$ and $a$.

\section{ACKNOWLEDGEMENTS}

The first author is partially supported by the Ministerio de Economía, Industria y Competitividad, Agencia Estatal de Investigación grants MTM2016-77278-P (FEDER), the Agència de Gestió d'Ajuts Universitaris i de Recerca grant 2017SGR1617, and the H2020 European Research Council grant MSCA-RISE-2017-777911. The second author is partially supported by FCT/Portugal through UID/MAT/04459/2013.

\section{REFERENCES}

[1] P. Acosta-Humánez, M. Alvarez-Ramirez and T.J. Stuchi, Nonintegrability of the Armbruster Guckenheimer Kim quartic Hamiltonian trough Morales Ramis theory, SIAM J. Appl. Dyn. Syst. 17 (2017), 78-96.

[2] D. Armbruster, J. GuCKenheimer And S. Kim, Chaotic dynamics in systems with square symmetry, Phys. Lett. A 140 (1989), 416-420.

[3] F. Dumortier, J. Llibre And J. C. Artés, Qualitative Theory of Planar Differential Systems, UniversiText, Springer-Verlag, New York, 2006.

[4] A.A. Elmandouh, On the dynamics of Armbruster Guckenheimer Kim galactic potential in a rotating reference frame, Astrophys. Space Sci. 361 (2016), 182-194.

[5] F.M. El-Sabaa, M. Hosny and S.K. Zakria, Bifurcations of Armbruster Guckenheimer Kim galactic potential, Astrophys. Space Sci. 364 (2019), 3443.

[6] M.W. HiRsch, Differential Topology, Graduate Texts in Mathematics, Springer, 1976.

[7] J. Llibre AND L. RoBerto, Periodic orbits and non-integrability of Armbruster-Guckenheimer-Kim potential, Astrophys. Space Sci. 343 (2012), 69-74. 


\begin{tabular}{|c|c|c|c|}
\hline$a$ & $h_{1}$ & $h_{2}$ & $I_{h_{1} h_{2}}$ \\
\hline$\leq 0$ & $<0$ & $\in \mathbb{R}$ & $\emptyset$ \\
\hline$\leq 0$ & $=0$ & $<0$ & $\phi$ \\
\hline$\leq 0$ & $=0$ & $=0$ & $\{(0,0)\} \times\{(0,0)\}$ \\
\hline$\leq 0$ & $=0$ & $>0$ & $\{(0,0)\} \times \mathbb{S}^{1}$ \\
\hline$\leq 0$ & $>0$ & $<0$ & $\emptyset$ \\
\hline$\leq 0$ & $>0$ & $=0$ & $\mathbb{S}^{1} \times\{(0,0)\}$ \\
\hline$\leq 0$ & $>0$ & $>0$ & $\mathbb{S}^{1} \times \mathbb{S}^{1}$ \\
\hline$\leq 0$ & $>1 /(4 a)$ & $>1 /(4 a)$ & $(\mathbb{R} \cup \mathbb{R}) \times(\mathbb{R} \cup \mathbb{R})$ \\
\hline$\leq 0$ & $>1 /(4 a)$ & $=1 /(4 a)$ & $(\mathbb{R} \cup \mathbb{R}) \times P$ \\
\hline$\leq 0$ & $>1 /(4 a)$ & $\in(0,1 /(4 a))$ & $(\mathbb{R} \cup \mathbb{R}) \times\left(\mathbb{R} \cup \mathbb{S}^{1} \cup \mathbb{R}\right)$ \\
\hline$\leq 0$ & $>1 /(4 a)$ & $=0$ & $(\mathbb{R} \cup \mathbb{R}) \times(\mathbb{R} \cup\{(0,0)\} \cup \mathbb{R})$ \\
\hline$\leq 0$ & $>1 /(4 a)$ & $<0$ & $(\mathbb{R} \cup \mathbb{R}) \times(\mathbb{R} \cup \mathbb{R})$ \\
\hline$\leq 0$ & $=1 /(4 a)$ & $>1 /(4 a)$ & $P \times(\mathbb{R} \cup \mathbb{R})$ \\
\hline$\leq 0$ & $=1 /(4 a)$ & $=1 /(4 a)$ & $P \times P$ \\
\hline$\leq 0$ & $=1 /(4 a)$ & $\in(0,1 /(4 a))$ & $P \times\left(\mathbb{R} \cup \mathbb{S}^{1} \cup \mathbb{R}\right)$ \\
\hline$\leq 0$ & $=1 /(4 a)$ & $=0$ & $P \times(\mathbb{R} \cup\{(0,0)\} \cup \mathbb{R})$ \\
\hline$\leq 0$ & $=1 /(4 a)$ & $<0$ & $P \times(\mathbb{R} \cup \mathbb{R})$ \\
\hline$\leq 0$ & $\in(0,1 /(4 a))$ & $>1 /(4 a)$ & $\left(\mathbb{R} \cup \mathbb{S}^{1} \cup \mathbb{R}\right) \times(\mathbb{R} \cup \mathbb{R})$ \\
\hline$\leq 0$ & $\in(0,1 /(4 a))$ & $=1 /(4 a)$ & $\left(\mathbb{R} \cup \mathbb{S}^{1} \cup \mathbb{R}\right) \times P$ \\
\hline$\leq 0$ & $\in(0,1 /(4 a))$ & $\in(0,1 /(4 a))$ & $\left(\mathbb{R} \cup \mathbb{S}^{1} \cup \mathbb{R}\right) \times\left(\mathbb{R} \cup \mathbb{S}^{1} \cup \mathbb{R}\right)$ \\
\hline$\leq 0$ & $\in(0,1 /(4 a))$ & $=0$ & $\left(\mathbb{R} \cup \mathbb{S}^{1} \cup \mathbb{R}\right) \times(\mathbb{R} \cup\{(0,0)\} \cup \mathbb{R})$ \\
\hline$\leq 0$ & $\in(0,1 /(4 a))$ & $<0$ & $\left(\mathbb{R} \cup \mathbb{S}^{1} \cup \mathbb{R}\right) \times(\mathbb{R} \cup \mathbb{R})$ \\
\hline$\leq 0$ & $=0$ & $>1 /(4 a)$ & $(\mathbb{R} \cup\{(0,0)\} \cup \mathbb{R}) \times(\mathbb{R} \cup \mathbb{R})$ \\
\hline$\leq 0$ & $=0$ & $=1 /(4 a)$ & $(\mathbb{R} \cup\{(0,0)\} \cup \mathbb{R}) \times P$ \\
\hline$\leq 0$ & $=0$ & $\in(0,1 /(4 a))$ & $(\mathbb{R} \cup\{(0,0)\} \cup \mathbb{R}) \times\left(\mathbb{R} \cup \mathbb{S}^{1} \cup \mathbb{R}\right)$ \\
\hline$\leq 0$ & $=0$ & $=0$ & $(\mathbb{R} \cup\{(0,0)\} \cup \mathbb{R}) \times(\mathbb{R} \cup\{(0,0)\} \cup \mathbb{R})$ \\
\hline$\leq 0$ & $=0$ & $<0$ & $(\mathbb{R} \cup\{(0,0)\} \cup \mathbb{R}) \times(\mathbb{R} \cup \mathbb{R})$ \\
\hline$\leq 0$ & $<0$ & $>1 /(4 a)$ & $(\mathbb{R} \cup \mathbb{R}) \times(\mathbb{R} \cup \mathbb{R})$ \\
\hline$\leq 0$ & $<0$ & $=1 /(4 a)$ & $(\mathbb{R} \cup \mathbb{R}) \times P$ \\
\hline$\leq 0$ & $<0$ & $\in(0,1 /(4 a))$ & $(\mathbb{R} \cup \mathbb{R}) \times\left(\mathbb{R} \cup \mathbb{S}^{1} \cup \mathbb{R}\right)$ \\
\hline$\leq 0$ & $<0$ & $=0$ & $(\mathbb{R} \cup \mathbb{R}) \times(\mathbb{R} \cup\{(0,0)\} \cup \mathbb{R})$ \\
\hline$\leq 0$ & $<0$ & $<0$ & $(\mathbb{R} \cup \mathbb{R}) \times(\mathbb{R} \cup \mathbb{R})$ \\
\hline
\end{tabular}

TABLE 2. The invariant sets $I_{h_{3} h_{4}}$ for the different values of $h_{3}, h_{4}$ and $a$

[8] T. Zeeuw And D. Merritt, Stellar orbits in a triaxial galaxy I. Orbits in the plane of rotation, Astrophys. 267 (1983), 571-595. 
Departament de Matemàtiques, Universitat Autònoma de Barcelona, 08193 Bellaterra, Barcelona, Catalonia, Spain

Email address: jllibre@mat.uab.cat

Departamento de Matemática, Instituto Superior Técnico, UniverSidAde De LisboA, 1049-001 LisboA, Portugal

Email address: cvalls@math.tecnico.ulisboa.pt 